\title{
NECESSARY AND SUFFICIENT CONDITIONS FOR $L_{1}$-STRONG- WEAK LOWER SEMICONTINUITY OF INTEGRAL FUNCTIONALS
}

\author{
ERIK J. BALDER \\ Mathematical Institute, University of Utrecht, Budapestlaan $6.3584 \mathrm{CD}$ Utrecht. Netherlands
}

(Received 27 April 1986; received for publication 27 October 1986)

Key words and phrases: Integral functionals, lower semicontinuity, seminormality.

\section{INTRODUCTION}

LET $(T, \mathscr{T}, \mu)$ BE a finite nonatomic measure space, $(X,\|\cdot\|)$ a separable Banach space, and $(V,|\cdot|)$ a separable reflexive Banach space, whose dual we denote by $V^{\prime}$. Let $f: T \times X \times V \rightarrow(-\infty,-x]$ be a given $\mathscr{T} \times \mathscr{B}(X \times V)$-measurable function. The associated integral functional $I_{f}: L_{X}^{\prime} \times L_{V} \rightarrow[-\infty,+\infty]$ is defined by

$$
I_{f}(x, v) \equiv \int_{T} f(t, x,(t), v(t)) \mu(\mathrm{d} t),
$$

where we use the convention [2, VII-7] regarding the possibly infinite values of the integral (this convention is compatible with the usual definition of outer integrals). We equip $L_{X}^{\prime}$ with the $L_{1}$-norm $\|\cdot\|_{1}$, and $L_{V}^{\frac{1}{1}}$ with the weak topology $\sigma\left(L_{V}^{\frac{1}{V}}, L_{V}^{*}\right)$, and are interested in necessary and sufficient conditions for strong-weak lower semicontinuity of $I_{f}$ on $L_{X}^{1} \times L_{V}^{1}$. The purpose of this note is to show how the seminormality approach of Balder [1] to the combined question of formulating necessary and sufficient conditions for lower semicontinuity of integral functionals extends to the present setting. For some details about seminormality we refer the reader to the Appendix and to [1]. The seminormality approach of [1] is based on the novel insight that, under quite general conditions, seminormality of the functions $f(t, \cdot, \cdot)$ on $X \times V$ $\mu$-a.e. and seminormality of $I_{f}$ on the Cartesian product of decomposable subspaces of $L_{X}^{0}$ and $L_{V}^{1}$ are equivalent. Here $L_{X}^{0}$ denotes the set of all (equivalence classes of) $(J, \mathscr{B}(X)$ )measurable functions from $T$ into $X$, equipped with the essential supremum norm. Now $L_{X}^{\frac{1}{X}}$ is certainly a decomposable subspace of $L_{X}^{0}$, but, in the context of [1], it still inherits the essential supremum topology (which is essential for the central equivalence result of [1] to hold). Moreover, the lower bounds for the integrand $f$ required in [1] are rather strict. Nevertheless, it will be made clear below that the equivalent seminormality results of [1] implies the main result of this note in a direct way.

\section{MAIN RESULT}

The main result of this note is as follows. 
THEOREM 2.1. The following three conditions

$$
\begin{aligned}
& f(t, \cdot, \cdot) \text { is sequentially l.s.c. on } X \times V \mu \text {-a.e., } \\
& f(t, x, \cdot) \text { is convex on } V \text { for every } x \in X \mu \text {-a.e., }
\end{aligned}
$$

there exist $M>0$ and $\psi \in L_{P}^{1}$ such that

$$
f(t, x, v) \geqslant \psi(t)-M(\|x\|+|v|) \quad \text { for all } x \in X, v \in V \mu \text {-a.e. }
$$

are sufficient for sequential strong-weak lower semicontinuity of $I_{f}$ on $L_{X}^{1} \times L_{V}^{1}$. Moreover, they are also necessary, provided that $I_{f}(\bar{x}, \bar{v})<+\infty$ for some $\bar{x} \in L_{X}^{1}, \bar{v} \in L_{V}^{1}$.

To prove this result, we shall use the main result theorem 3.6 of [1]. Specialized to the framework of this note, it runs as follows (note in particular that $X$ is a Suslin space and that $L_{X}^{l}$ is a decomposable subspace of $L_{X}^{0}$; see also example 4.2 of [1]).

Theorem (EQUiVAlent SEMINORMality). Suppose that $l: T \times X \times V \rightarrow(-\infty,+\infty)$ is a $\mathscr{T} \times \mathscr{B}(X \times V)$-measurable function for which there exist $p \in L_{V^{\prime}}^{x}$ and $\phi \in L_{\mathbb{R}}^{1}$ such that

$$
l(t, x, v) \geqslant\langle v, p(t)\rangle+\phi(t) \text { for all } x \in X, v \in V \mu \text {-a.e. }
$$

Then the condition

$$
l(t, \cdot, \cdot) \text { is seminormal on } X \times V \mu \text {-a.e. }
$$

is sufficient for the seminormality of $I_{l}$ on $L_{X}^{1} \times L_{V}^{1}$. Moreover, it is also necessary, provided that $I_{l}(\bar{x}, \bar{v})<+\infty$ for some $\bar{x} \in L_{X}^{1}, \bar{v} \in L_{V}^{1}$.

The proof of this equivalence result in [1] strongly depends upon the possibility to reformulate seminormality-which is a generalized convexity property-in terms of Fenchel-like conjugation. After this, one can use the measurable selection apparatus developed for the conjugation of integral functionals, which is mainly due to R. T. Rockafellar [8], [2, VII].

Proof of theorem 2.1. The proof of sufficiency of (2.1)-(2.3) by means of the equivalence theorem is not new; it can be found in [1, Section 4], where e.g. corollary 4.11 immediately implies this result. Nevertheless, for the sake of coherence we also describe a proof of sufficiency here; cf. [1, Section 4] for some details.

Suppose that $\left\{x_{k}\right\}_{0}^{x} \subset L_{X}^{1}$ and $\left\{v_{k}\right\}_{0}^{x} \subset L_{V}^{1}$ are sequences with $x_{k} \rightarrow x_{0}$ strongly and $v_{k} \rightarrow v_{0}$ weakly. Rather than selecting suitable sequences and invoking Egorov's theorem, we can suppose without loss of generality that $x_{k} \rightarrow x_{0}$ in the essential supremum norm. Since $\left\{v_{k}\right\}_{0}^{\infty}$ is relatively sequentially compact, it follows by the Dunford-Pettis theorem [4, IV.2.1] that $\left\{\left\|v_{k}(\cdot)\right\|\right\}_{0}^{\infty}$ is uniformly integrable. Hence, by the theorem of de la Vallée Poussin, there exists a nondecreasing continuous convex function $h^{\prime}:[0,+\infty) \rightarrow[0,+\infty)$ such that

$$
\begin{aligned}
& \lim _{\gamma \rightarrow x} h^{\prime}(\gamma) / \gamma=+\infty \\
& \sigma \equiv \sup _{k} \int_{T} h^{\prime}\left(\left\|v_{k}(t)\right\|\right) \mu(\mathrm{d} t)<+\infty .
\end{aligned}
$$

Define $h: V \rightarrow[0,+\infty)$ by setting $h(v) \equiv h^{\prime}(\|v\|)$; then $h$ is inf-compact for every slope (by 
(2.4) and reflexivity of $V)$ and convex on $V$. For $\varepsilon>0$ we define $f_{\varepsilon}: T \times X \times V \rightarrow(-x,+\infty]$ by

$$
f_{\varepsilon}(t, x, v) \equiv f(t, x, v)+M\|x\|+\frac{1}{2} \varepsilon h(v) .
$$

By (2.3)-(2.4) and nonnegativity of $h^{\prime}$. there exists for every $\varepsilon>0$ a constant $C_{\varepsilon}$ such that

$$
f_{\varepsilon}(t, x, v) \geqslant \psi(t)+C_{\varepsilon} .
$$

Note also that for every $\varepsilon>0$

$$
\begin{aligned}
& f_{\varepsilon}(t, \cdot, \cdot) \text { is sequentially l.s.c. on } X \times V \mu \text {-a.e., } \\
& f_{\varepsilon}(t, x, \cdot) \text { is convex on } V \text { for every } x \in X \mu \text {-a.e. }
\end{aligned}
$$

As a consequence of the above, by the proposition of the Appendix, the function $l_{\varepsilon}: T \times X \times V \rightarrow(-\infty,+\infty]$, defined by

$$
l_{\varepsilon}(t, x, v) \equiv f_{\varepsilon}(t, x, v)+\frac{1}{2} \varepsilon h(v),
$$

is such that

$$
l_{\varepsilon}(t, \cdot, \cdot) \text { is seminormal on } X \times V \mu \text {-a.e. }
$$

for every $\varepsilon>0$. In view of (2.6), the sufficiency part of the equivalence theorem now gives that for every $\varepsilon>0$

$$
I_{l_{\varepsilon}}=I_{f}+M\|\cdot\|_{1}+\varepsilon I_{h}
$$

is seminormal on $L_{X}^{1} \times L_{V}^{1}$. Since clearly by virtue of (2.5)

$$
\sup _{\varepsilon>0}\left(I_{l_{\varepsilon}}\left(x_{k}, v_{k}\right)-\varepsilon \sigma\right)=I_{f}\left(x_{k}, v_{k}\right)+M\left\|x_{k}\right\|_{1},
$$

it now follows immediately that

$$
\liminf _{k \rightarrow \infty} I_{f}\left(x_{k}, v_{k}\right) \geqslant I_{f}\left(x_{0}, v_{0}\right)
$$

which finishes the proof of the sufficiency part.

For reasons explained in the introduction, the proof of necessity does not directly follow from any result in [1]; however, we shall give an argument here that closely resembles the proof of theorem 4.12 in [1].

As for necessity of (2.3), we can repeat the simple argument given in [6]: if (2.3) does not hold for any $M>0$, there exist for every $M \in \mathbb{N}$ a set $A_{M}$ in $\mathscr{T}$ and a function $\phi_{M}$ in $L_{R}^{1}$ such that $M \mu\left(A_{M}\right)=1$ and

$$
\begin{aligned}
& \int_{A_{M}} \phi_{M}(t) \mu(\mathrm{d} t)=-1 \\
& 0 \geqslant \phi_{M}(t) \geqslant \inf _{x \in X, v \in V}(f(t, x, v)+M|| x \|+M|v|)+1,
\end{aligned}
$$

where the nonatomicity of $\mu$ has been used. By the von Neumann-Aumann measurable selection theorem, there exist for every $M \in \mathbb{N}$ functions $x_{M} \in L_{X}^{0}, v_{M} \in L_{V}^{0}$ such that

$$
f\left(t, x_{M}(t), v_{M}(t)\right)+M\left(\left\|x_{M}(t)\right\|+\left|v_{M}(t)\right|\right) \leqslant \phi_{M}(t) \mu \text {-a.e. }
$$

For every $M \in \mathbb{N}$ there exist $\tilde{A}_{m} \in \mathcal{T}, \tilde{A}_{M} \subset A_{M}$, such that

$$
M \int_{\hat{A}_{m}}\left(\left\|x_{M}(t)\right\|+\left|v_{M}(t)\right|\right) \mu(\mathrm{d} t)=1 .
$$

We define $\bar{x}_{M} \in L_{X}^{\prime}$ and $\bar{v}_{M} \in L_{V}^{1}$ by setting $\left(\tilde{x}_{M}, \bar{v}_{M}\right) \equiv\left(x_{M}, v_{M}\right)$ on $\bar{A}_{M}$ and $\left(\tilde{x}_{M}, \tilde{v}_{M}\right) \equiv(\bar{x}, \bar{v})$ 
on $T \backslash \bar{A}_{M}$. By (2.7) $x_{M} \rightarrow \bar{x}$ strongly in $L_{X}^{1}$ and $v_{M} \rightarrow \bar{v}$ strongly in $L_{V}^{1}$. By (2.6), (2.7) we have

$$
\liminf _{u \rightarrow x} I_{f}\left(\dot{x}_{. M}, \bar{v}_{. H}\right) \leqslant I_{f}(\vec{x}, \bar{v})-1,
$$

so a contradiction with the sequential lower semicontinuity of $I_{f}$ at $(\bar{x}, \bar{v})$ has been reached. We conclude that (2.3) holds.

We now prove (2.1) and (2.2) by means of the necessity part of the equivalent seminormality theorem. Since $\{\bar{v}\}$ is compact in $L_{V}$, it follows by the Dunford-Pettis theorem and the de la Vallée Poussin theorem that there exists $h^{\prime}:[0,+\infty) \rightarrow[0,+\infty)$ having the same properties as stated in the sufficiency proof, with (2.4) and

$$
I_{h}(\bar{v})<+\infty,
$$

where $h$ corresponds to $h^{\prime}$ just as above. By the Dunford-Pettis theorem $I_{h}$ is inf-compact on $L_{V}^{1}$ for every slope; of course, it is also l.s.c. and convex on $L_{V}^{1}$. For every $\varepsilon>0$ we define $f_{\varepsilon}$ just as above; evidently,

$$
I_{f_{\varepsilon}} \text { is sequentially l.s.c. on } L_{X}^{1} \times L_{V}^{1} \text {. }
$$

Also, for every $x \in L_{X}^{1}$ the functional $I_{f_{\varepsilon}}(x, \cdot)$ is sequentially l.s.c. on $L_{V} \cdot$. Since it is minorized by the integral functional

$$
J_{\varepsilon}(v) \equiv \int_{T}\left(\frac{1}{2} \varepsilon h^{\prime}(|v(t)|)-M|v(t)|\right) \mu(\mathrm{d} t) .
$$

whose level sets are relatively compact by the Dunford-Pettis theorem, it follows from the Eberlein-Šmulian theorem that for every $\varepsilon>0$

$$
I_{f_{\varepsilon}}(x, \cdot) \text { is l.s.c. on } L_{V}^{1} \text { for every } x \in L_{X}^{1} .
$$

A well-known consequence of this is that for every $\varepsilon>0$

$$
I_{f_{\varepsilon}}(x, \cdot) \text { is convex on } L_{V}^{\prime} \text { for every } x \in L_{X}^{1},
$$

by Lyapunov's theorem (e.g., see the proof of [1, theorem 4.12] for details). Since (2.3) and (2.4) have been established, (2.6) holds again for $f_{\varepsilon}$. Thus, for every $\varepsilon>0$

$$
I_{f_{\varepsilon}}(x, v) \geqslant \int_{T} \psi \mathrm{d} \mu+C_{\varepsilon} \mu(T) .
$$

We may now apply the proposition of the Appendix. This gives that for every $\varepsilon>0$

$$
I_{l_{s}}=I_{f_{s}}+\frac{1}{2} \varepsilon I_{h} \text { is seminormal on } L_{X}^{1} \times L_{V}^{1} \text {. }
$$

Here $l_{\varepsilon}$ corresponds to $f$ and $h$ as before: $l_{\varepsilon} \equiv f+M\|\cdot\|+\varepsilon h$. We can now apply the necessity part of the equivalent seminormality theorem, since also

$$
I_{l_{\varepsilon}}(\bar{x}, \bar{v})<+x
$$

(valid by $\left(2.5^{\prime}\right)$ ). This gives that for every $\varepsilon>0$

$$
l_{\varepsilon}(t, \cdot, \cdot) \text { is seminormal on } X \times V \mu \text {-a.e. }
$$

Letting $\varepsilon \rightarrow 0$ it is now easy to establish that (2.1)-(2.2) must hold. 
Theorem 2.1 extends the main result of Olech [6], where $X$ and $V$ were finite-dimensional. As is the case in all approaches by others that are known to the author, the proofs of necessity and sufficiency in [6] differ widely. While it is not unusual to see the use of seminormality in the sufficiency part of similar theorems (the work of Tonelli [9] and Cesari [3] shows this in abundance), seminormality has not played a role (before [1]) in the proofs of necessary conditions for lower semicontinuity. An earlier necessity result is due to Polyak [7]: we refer to $[3,10.9]$ and $[5]$ for details.

It is well-known that the nice, clean characterization of sequential strong-weak lower semicontinuity of [6] and the present note no longer hold for $L_{p}$-spaces with $p>1$. Some counterexamples can be found in [5, pp. 525-526]. In terms of our proof this failure can be understood by realizing that, while the lower bounds for the integrand would necessarily be of the type $\psi(t)-M\left(\|x\|^{p}+|v|^{p}\right)$, the functions $h^{\prime}$ of our proof would have to be of the type $h^{\prime}(\gamma)=\gamma^{p}$. Hence, such instrumental functions as $f_{\varepsilon}=f+M\|\cdot\| p+\frac{1}{2} \varepsilon h$ and $l_{\varepsilon}=$ $f+M\|\cdot\| p+\varepsilon h$ would no longer satisfy the boundedness and growth conditions needed to obtain seminormality, unless additional assumptions were introduced.

\section{REFERENCES}

1. BALder E. J., On seminormality of integral functionals and their integrands, SIAM J. Control Optim. 24 95-121 (1986).

2. CASTAING C. \& VAI.ADIER M., Convex analysis and measurable multifunctions, Lecture Notes in . Wathematics 580, Springer, Berlin (1977).

3. CESARI L., Optimization-Theory and Applications, Problems with Ordinary Differential Equations, Springer, Berlin (1983).

4. Diestel. J. \& Uhl J. J., Vector measures. Mathematical Surveys 15, American Mathematical Society, Providence (1977).

5. IOFFE A. D., On lower semicontinuity of integral functionals I, SIAM J. Control Optim. 15, 521-538 (1977).

6. OLECH C., A characterization of $L_{1}$-weak lower semicontinuity of integral functionals, Bull. Acad. pol. Sci. Sér. Sci. math. astr. phys. 25, 135-142 (1977).

7. Poljak B. T., Semicontinuity of integral functionals and existence theorems, Mat. Sb. 78. 65-84 (1969); Math. U.S.S.R. Sb. 7, 59-77 (1969).

8. Rockafellar R. T., Integrals which are convex functionals, Pacif. J. Math. 24, 525-539 (1969).

9. TONelLi L., Sugli integrali del calcolo delle variazioni in forma ordinaria, Ann. Scu. norm. sup. Pisa 2(3), 401450 (1934).

10. Nougues-SaINTE-Beuve M. F., Conditions nécessaires pour la semi-continuité inférieure d'une fonctionelle intégrale, Seminaire d'Analyse Convexe, Université des Sciences et Techniques du Languedoc 15. 10.1-10.36 (1985).

\section{APPENDIX}

Let $(Y, d)$ be a metric space and $(W, P)$ a pair of Hausdorff locally convex topological vector spaces. paired by a duality $(\cdot, \cdot)$. According to [1], a function $a: Y \times W \rightarrow[-\infty,+\infty]$ is said to be simple seminormal on $Y \times W$ if

$$
a(y, w)=g(y)+\langle w, p\rangle
$$

for some 1.s.c. function $g: Y \rightarrow[-x,+x]$ and some $p \in P$. Further, $a$ is said to be seminormal on $Y \times W$ if $a$ is the pointwise supremum of a collection of simple seminormal functions on $Y \times M$. This definition goes back to Tonelli $[9]$ and is strongly related to Cesari's property $(Q)$ for multifunctions; cf. [1]. Recall that a function $h: W \rightarrow(-\infty$, $+\infty]$ is said to be inf-compact on $W$ for every slope if for every $p \in P$ the set $\{w \in W: h(w)-\langle w, p\rangle \leqslant \beta\}$ is compact for every $\beta \in \mathbb{R}$. The following criterion for seminormality goes back to Tonelli; cf. [1, corollary 2.9].

Proposition. Suppose that sequential compactness and compactness on $W$ are equivalent. Suppose that the function a: $Y \times W \rightarrow[-\infty,+\infty]$ satisfies the following conditions:

$$
\begin{aligned}
& a \text { is sequentially l.s.c. on } Y \times W ; \\
& a(y, \cdot) \text { is convex on } W \text { for every } y \in Y ;
\end{aligned}
$$




$$
\begin{aligned}
& \text { there exist } p \in P \text { and } \beta \in R \text { such that } \\
& a(y, w) \geqslant\langle w, p\rangle+\beta \quad \text { for all } \quad y \in Y, w \in W .
\end{aligned}
$$

Then for every $h: W \rightarrow(-\infty,+x)$ which is convex and inf-compact on $W$ for every slope, the function $a_{\varepsilon}$, defined by

$$
a_{\varepsilon}(y, w) \equiv a(y, w)+\varepsilon h(w)
$$

is seminormal on $Y \times W$ for every $\varepsilon>0$.

For the applications of this result in this note we abserve that both on $V$ and $L_{v}^{1}$, equipped with their weak topologies, sequential and topological compactness are equivalent by the Eberlein-Šmulian theorem. 